\title{
The Second LAT AGN Catalogue from the Fermi observatory
}

\section{Sara Cutini*}

Agenzia Spaziale Italiana (ASI) Science Data Center

E-mail: sara.cutini@asdc.asi.it

\section{Elisabetta Cavazzuti}

Agenzia Spaziale Italiana (ASI) Science Data Center

E-mail: elisabetta.cavazzuti@asdc.asi.it

\section{Charles Dermer}

Space Science Division, Naval Research Laboratory, Washington, DC 20375-5352

E-mail: charles.dermer@nrl.navy.mil

\section{Dario Gasparrini}

Agenzia Spaziale Italiana (ASI) Science Data Center

E-mail: dario.gasparrini@asdc.asi.it

\section{Benoit Lott}

Université Bordeaux 1, CNRS/IN2p3, Centre d'Études Nucléaires de Bordeaux Gradignan, 33175 Gradignan, France

E-mail: lottecenbg.in2p3.fr

\section{on behalf the Fermi-LAT Collaboration}

The second catalog of active galactic nuclei (AGNs) detected by the Fermi Large Area Telescope (LAT) in two years of scientific operation is presented. The Second LAT AGN Catalog (2LAC) includes $1017 \gamma$-ray sources located at high Galactic latitudes $(a b s(b)>10 \mathrm{deg})$ that are detected with a test statistic (TS) greater than 25 and associated statistically with AGNs. Consequently we define a Clean sample which includes 886 AGNs, comprising 395 BL Lacertae objects (BL Lacs), 310 flat-spectrum radio quasars (FSRQs), 157 candidate blazars of unknown type (i.e., with broad-band blazar characteristics but with no optical spectral measurement yet), eight misaligned AGNs, four narrow-line Seyfert 1 (NLS1s), 10 AGNs of other types and two starburst galaxies. The full characterization of the newly detected sources will require more broad-band data. Various properties, such as $\gamma$-ray fluxes and photon power law spectral indices, redshifts, $\gamma$-ray luminosities, variability, and archival radio luminosities and their correlations are presented and discussed for the different blazar classes. The general trends observed in 1LAC are confirmed.

The Extreme and Variable High Energy Sky

September 19-23, 2011

Chia Laguna (Cagliari), Italy

*Speaker. 


\section{Source Association Methods and Classification}

The LAT localization accuracy is not precise enough to permit the determination of a lower energy counterpart based only on positional coincidence. We assert a firm counterpart identification only if the variability detected by the LAT corresponds with variability at other wavelengths. In order to improve over the results of 1LAC by including these faint radio sources, the association procedure for building the 2LAC list makes use of three different methods: the Bayesian Method (used in 1FGL/1LAC) and two additional methods, namely the Likelihood Ratio Method and the $\log N \log S$ Method. For a counterpart to be considered as associated, its association probability must be $>0.8$ for at least one method. The two additional methods improve the association results through the use of physical properties of the candidate counterparts, such as the surface density and the spectral shape in the radio energy band, in addition to the positional coincidence with the $\gamma$-ray source. Considering potential counterparts with lower radio flux enables more highsynchrotron peaked BL Lacs to be selected but the number of FSRQs is also increased. This is achieved through the use of surveys and serendipitous findings, as the available catalogs (used by the Bayesian Method) are not deep enough. The ingredients of the classification procedure are optical spectrum or other blazar characteristics.

To classify a source optically we made use of, in decreasing order of precedence: optical spectra from our intensive follow-up programs, the BZCAT list (i.e., FSRQs and BL Lacs in this list), spectra available in the literature. If an optical spectrum was not available, we looked for the evidence of typical blazar characteristics, such as radio loudness, a flat radio spectrum at least between $1.4 \mathrm{GHz}$ and $5 \mathrm{GHz}$, broad band emission. We defined the classes as follow:

- FSRQ, BL Lac, radio galaxy, steep-spectrum radio quasar (SSRQ), Seyfert, NLS1, starburst galaxy for sources with well-established classes in literature and/or through an optical spectrum with a good evaluation of emission lines.

- AGU - for blazar-like sources without a good optical spectrum

- AGN - this class is more generic than AGU. These sources are not confirmed blazars.

\section{Source Properties}

\subsection{SED Classification}

As in 1LAC, we classify blazars also based on the synchrotron peak frequency of the broadband SED [Abdo et al. 2010]. This scheme extends to all blazars the standard classification system introduced by [Padovani \& Giommi 1995] for BL Lacs. We estimate the synchrotron peak frequency $v_{\text {peak }}^{S}$, using the broadband indices $\alpha_{r o}$ (between $5 \mathrm{GHz}$ and $5000 \AA$ ) and $\alpha_{o x}$ (between $5000 \AA$ and $1 \mathrm{keV}$ ). However, looking at the whole sample we can see that the two classes of objects have different distributions. For FSRQs, the average $\left\langle\log v_{\text {peak }}^{S}\right\rangle$ obtained in the $2 \mathrm{LAC}$ Clean Sample is $13.02 \pm 0.35$ while BL Lacs spread the whole parameter space from low (LSP) to the highest frequencies (HSP). These results are consistent with those presented in [Abdo et al. 2010] and in [Giommi et al. 2011]. The photon index is plotted versus the frequency of the synchrotron peak in Figure 1. A relatively strong correlation between these two parameters, again reported earlier [Abdo et al. 2009, Abdo et al. 2010] is observed. 


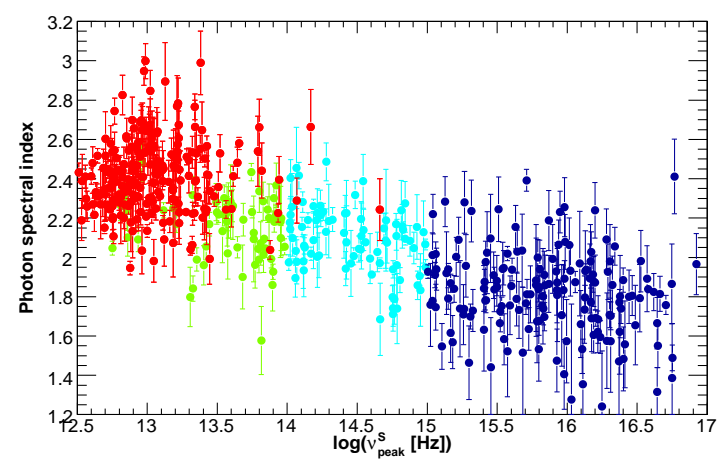

Figure 1: Photon index versus frequency of the synchrotron peak $v_{\text {peak }}^{S}$. Red: FSRQs, green: LSP-BL Lacs, light blue: ISP-BL Lacs, dark blue: HSP-BL Lacs.

\subsection{Non-Blazar Objects and Misaligned AGNs}

Non-blazar $\gamma$-ray AGN (those not classified as FSRQs, BL Lacs, or as AGU) constitutes a small fraction of sources in the $2 \mathrm{LAC}(\sim 3 \%)$. The main part of there AGN are radio galaxies, the 2LAC contains in particular two new radio galaxies: Centaurus B and Fornax A, associated to $2 F G L J 1346.6-6027$ and $2 F G L J 0322.4-3717$, respectively. The 2LAC also includes the detection of $2 F G L J 0316.6+4119$ associated with the head-tail radio galaxy IC 310 whose spectrum extends up to VHE. The emission from starburst-galaxy were already detected in the case of $M$ 82, NGC 253, NGC 1068 and NGC 4945 in the first year of operation and confirmed with better localization in the second one. The low-probability association of $1 F G L J 1307.0-4030$ with the nearby Seyfert galaxy ESO $323-G 77$ is confirmed with $2 F G L J 1306.9-4028$. No new radio-loud narrow-line Seyfert 1 galaxies beyond those four detected in the 1st year [Abdo et al. 2009].

\subsection{Flux and Photon Spectral Index Distributions}

The photon index is plotted versus the mean flux $(\mathrm{E}>100 \mathrm{MeV})$ in Figure 2, along with an estimate of the flux limit. The flux limit strongly depends on the photon index as harder sources are easier to discriminate against the background, which is due to the narrowing of the point-spread function (PSF) of the LAT with increasing energy and to the relative softness of the diffuse Galactic $\gamma$-ray emission. In contrast, the limit in energy flux above $100 \mathrm{MeV}$ is almost independent of the photon index as illustrated in Figure 2.

\subsection{Redshift Distributions}

The redshift distributions of the various classes are shown in Figure 3. The distribution peaks around $\mathrm{z}=1$ for FSRQs (Fig. 3 top) and extends to $\mathrm{z}=3.10$. The distribution peaks at a lower redshift for BL Lacs (Figure 3 middle). Note that $55 \%$ of the BL Lacs have no measured redshifts. The fraction of BL Lacs having a measured redshift is higher for sources with a SED-based classification. This fraction is essentially constant for the different subclasses, $(49 \%, 49 \%, 53 \%)$ for (LSPs, ISPs, HSPs) respectively. Figure 3 bottom shows the redshift distributions for the different subclasses of BL Lacs. These distributions gradually extend to lower redshifts as the location of the synchroton peak shifts to higher frequency, i.e., from LSPs to HSPs. 

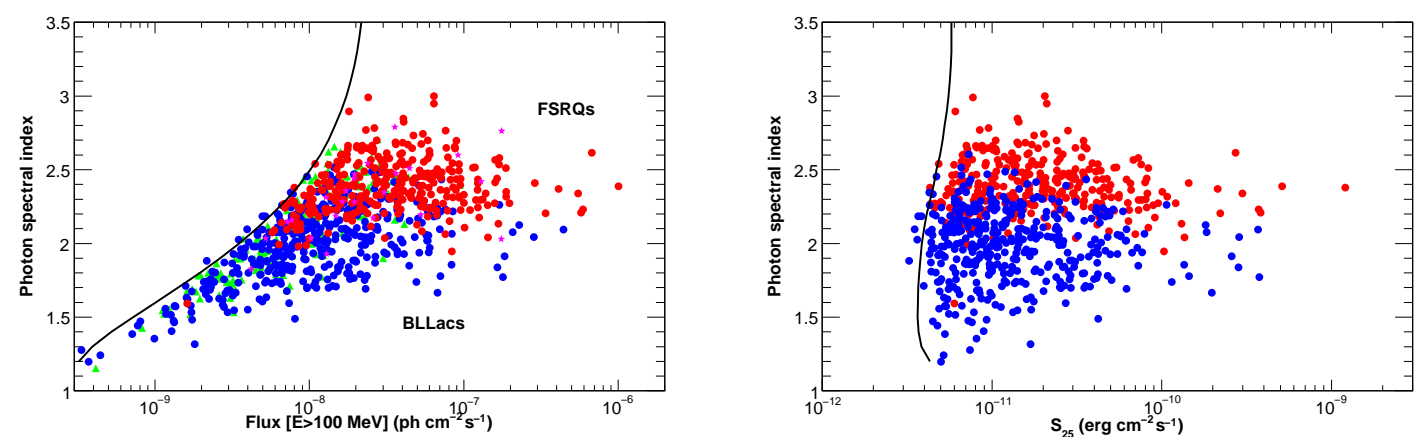

Figure 2: Photon index versus energy flux above $100 \mathrm{MeV}$. Red: FSRQs, blue: BL Lacs. The curve represents the approximate detection limit.
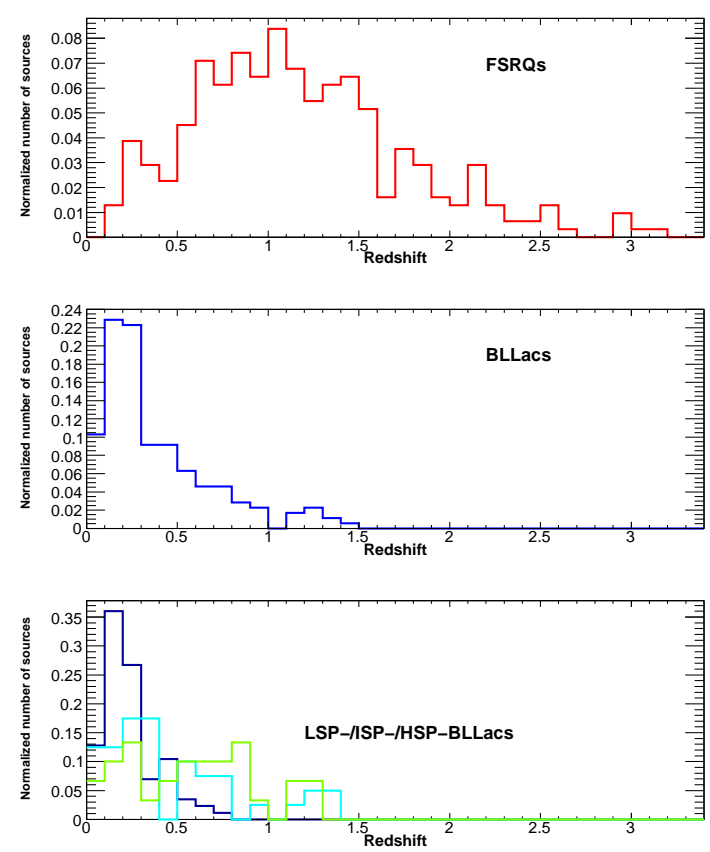

Figure 3: Redshift distributions for FSRQs (top), BL Lacs (middle), LSP-BL Lacs (bottom, green), ISPBL Lacs (bottom, light blue), HSP-BL Lacs (bottom, dark blue)

\subsection{Variability}

Variability at all time scales is one of the distinctive properties of blazars. Since launch, detections by the Fermi-LAT of $\gamma$-ray activity from 81 flaring blazars have been reported in Astronomer Telegrams (ATels). In Figure 4 we have plotted the average PDS for FSRQs and BL Lacs. The power density is normalized to fractional variance per frequency unit $\left(\mathrm{rms}^{2} \mathrm{I}^{-2} \mathrm{day}^{-1}\right.$, where $\mathrm{I}$ is the average flux) and the PDS points are averaged in logarithmic frequency bins. The white noise level was estimated from the rms of the flux errors and was subtracted for each PDS. The error bars were computed as the standard error of the mean for each frequency bin. The PDS slope (powerlaw index) is similar for the two groups, $\sim 1.15 \pm 0.10$. This is somewhat flatter than was deduced 


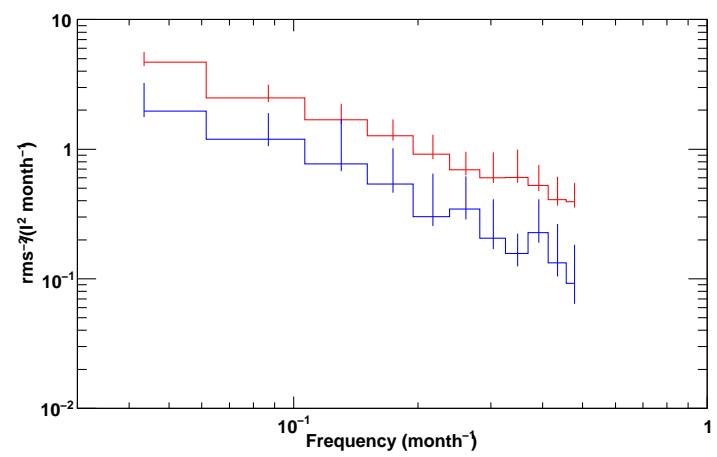

Figure 4: Power density spectrum (PDS) for bright FSRQs (red) and BL Lacs (blue).

for the very brightest sources in the LBAS sample [Abdo et al. 2010]. The difference in the height of the PDS means that the fractional variability of BL Lacs is lower than that of FSRQs. This is in line with the LBAS results.

\subsection{Radio properties}

The 2LAC sources are associated with a population of radio sources, whose flux density distribution spans the range between a few mJy and several tens of Jy. In Figure 5 we plot the radio flux density distributions for sources in the 2LAC, divided according to the optical type. The distributions for BL Lacs and FSRQs are quite broad, with well separated peaks, FSRQs being on average significantly brighter radio sources. The median flux densities of the two distributions at $8 \mathrm{GHz}$ are 86 and $581 \mathrm{mJy}$ for BL Lacs and FSRQ, respectively. The distributions are similar for the three frequencies $(8 \mathrm{GHz}, 20 \mathrm{GHz}, 30 \mathrm{GHz})$, confirming that the 2LAC sources have flat radio spectra. Below $1 \mathrm{Jy}$, Planck counts drop rapidly owing to sensitivity limits, while AT20G becomes less and less complete below $100 \mathrm{mJy}$. Interestingly, AT20G shows a deficit of BL Lac sources in the 100-300 mJy range, which cannot be attributed to sensitivity limits; this is most likely to arise from the lack of spectroscopic information for sources in the Southern hemisphere, where the AT20G survey was carried out.

\subsection{Highest-energy photons}

Figure 6 displays, as a function of redshift, the highest energy photon (HEP) detected by the LAT from the 2LAC AGN sample using the Pass 7_V6 Ultraclean event selection and that is associated with the source within the $68 \%$ containment radius. Predictions of $\gamma \gamma$ opacity curves $\tau_{\gamma \gamma}=3$ for different EBL models are also shown in Figure 6. Detection of HEPs above the opacity curve predicted by a given model makes the model less likely. In the new 2LAC AGN sample, we find 30 HEP events from $z>0.5$ sources beyond the $\tau_{\gamma \gamma}=3$ regime of the [Stecker et al. 2006 ] "baseline model", which is already severely constrained by the LAT 11 month data set [Abdo et al. 2010].

\section{Conclusions and Results}

The $2 \mathrm{LAC}$ represents a significant advance with respect to the $1 \mathrm{LAC}$, including many more sources and reduced uncertainties thanks to the doubling of exposure and refinement of the analy- 

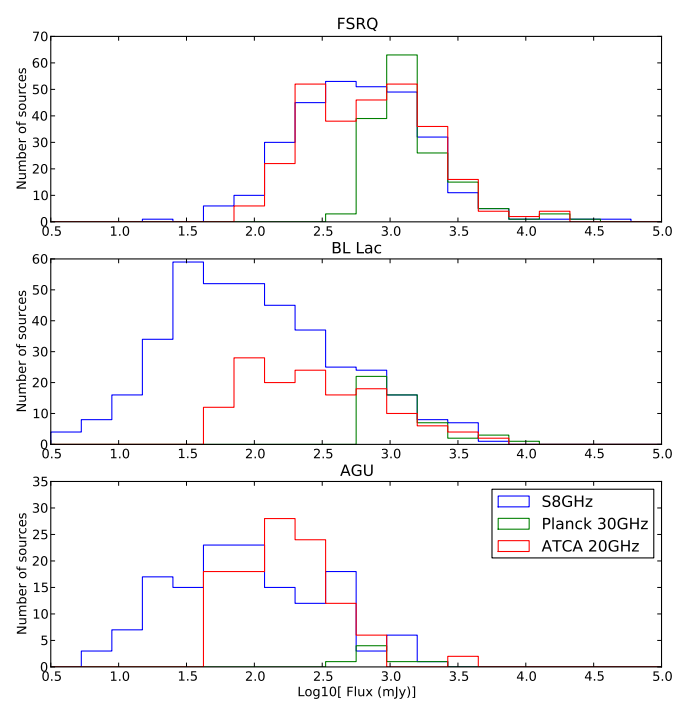

Figure 5: Radio flux density distributions of the 2LAC counterparts: FSRQs (top), BL Lacs (middle), blazars of unknown type (bottom). For each panel we show the counts at $8 \mathrm{GHz}$ (blue line, from CRATES or similar surveys), at $20 \mathrm{GHz}$ (red line, obtained from the AT20G survey and multiplied by 2 to normalize for the sky coverage) and at $30 \mathrm{GHz}$ (green line, from the Planck ERCSC).

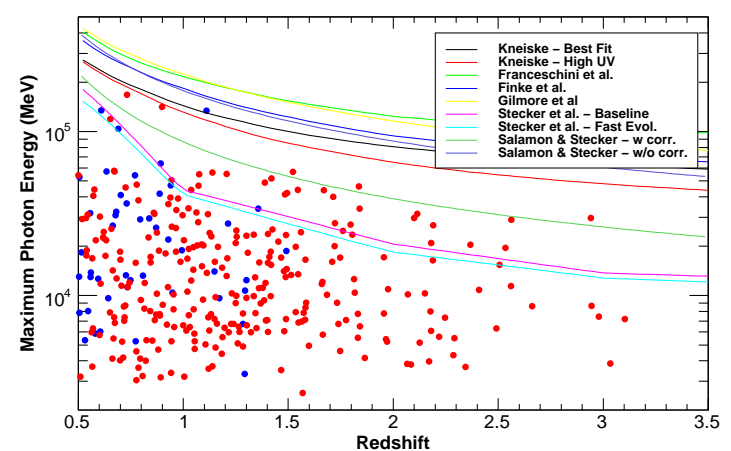

Figure 6: Maximum photon energy versus redshift. Red: FSRQs, blue: BL Lacs. The curves correspond to predictions for $\tau=3$ for different models.

sis. This has resulted in an $\sim 52 \%$ (1017 versus 671) increase in the number of associated sources, better localization, more accurate time-averaged spectra, and more detailed light curves and characterization of variability patterns. The general trends identified in the 1LAC, many of them already apparent in the LBAS, are confirmed.

\section{References}

[Abdo et al. 2010] ApJ, 723, 1082 (Extragalactic Background Light)

[Abdo et al. 2010] ApJ, 722, 520 (Variability of LBAS blazars) 
[Abdo et al. 2009] ApJ, 700, 597 (LBAS)

[Abdo et al. 2009] ApJ, 707, L142

[Ajello et al. 2009] ApJ, 699, 603

[Abdo et al. 2010] ApJ, 716, 30 (SEDs of bright Fermi blazars)

[Abdo et al. 2010] ApJ, 715, 429 (1LAC)

[Giommi et al. 1999] MNRAS, 310, 465

[Giommi et al. 2011] ArXiv:1108.1114

[Padovani \& Giommi 1995] ApJ, 444, 567

[Stecker et al. 2006] ApJ, 648, 774 